\title{
Prognostic predictors in pericardiectomy for chronic constrictive pericarditis
}

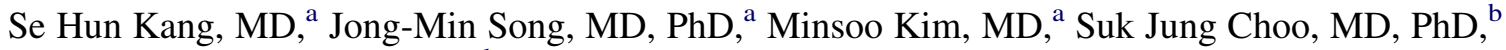 \\ Cheol Hyun Chung, MD, PhD, ${ }^{\mathrm{b}}$ Duk-Hyun Kang, MD, PhD, ${ }^{\mathrm{a}}$ and Jae-Kwan Song, MD, $\mathrm{PhD}^{\mathrm{a}}$
}

\begin{abstract}
Objective: Prognosis after pericardiectomy remains to be clearly elucidated, especially in Asian countries, where the causes of constrictive pericarditis differ from those in Western countries. We aimed to investigate the preoperative prognostic factors and clinical outcomes after pericardiectomy in patients with chronic constrictive pericarditis.
\end{abstract}

\begin{abstract}
Methods: Preoperative clinical and imaging characteristics were evaluated in 85 consecutive patients with chronic constrictive pericarditis without other valvular or ischemic heart diseases who underwent pericardiectomy. Causes were idiopathic in 49 patients $(57.6 \%)$ and tuberculous in 36 patients $(42.4 \%)$. All-cause death was observed for a median of 38.5 months.
\end{abstract}

Results: Of 85 patients, 15 (17.6\%) died during follow-up. These 15 patients who died during follow-up had higher aspartate aminotransferase, smaller left ventricular end-systolic dimension index, and higher early diastolic mitral inflow velocity before pericardiectomy than the 70 patients who survived. Multivariate Cox proportional analysis showed that diabetes mellitus (hazard ratio, 4.610; $P=.024$ ) and high early diastolic mitral inflow velocity (hazard ratio, $1.050 / \mathrm{cm} / \mathrm{s} ; P=.002$ ) before pericardiectomy were independent predictors of mortality after pericardiectomy. The preoperative cutoff value for early diastolic mitral inflow velocity in predicting mortality after pericardiectomy was $71 \mathrm{~cm} / \mathrm{s}$ (sensitivity of $84.6 \%$ and specificity of $52.2 \%$ ), and there was a significant difference in survival between groups divided by this cutoff value of early diastolic mitral inflow velocity $(P=.029)$.

Conclusions: Preoperative high early diastolic mitral inflow velocity and diabetes mellitus were predictors of poor prognosis after pericardiectomy in patients with chronic constrictive pericarditis. These results suggest that preoperative Doppler echocardiographic evaluation may be valuable not only for diagnosing constrictive pericarditis but also for predicting prognosis after pericardiectomy. (J Thorac Cardiovasc Surg 2014;147:598-605)

Constrictive pericarditis $(\mathrm{CP})$ is a disease characterized by impaired diastolic ventricular filling resulting from a constriction caused by scarred fibrotic pericardium. ${ }^{1}$ The development of noninvasive imaging techniques, including Doppler echocardiography, has facilitated early clinical recognition of CP. CP is usually a long-term consequence of acute or chronic forms of pericarditis, which may be caused by infectious diseases such as tuberculosis, viruses, and bacteria; by malignancies; and by complications associated with cardiac surgery and radiation therapy.

Pericardiectomy is the definitive therapy for $\mathrm{CP}$, resulting in complete relief of symptoms in many patients. ${ }^{1-3}$ However,

From the Division of Cardiology ${ }^{\mathrm{a}}$ and Department of Cardiovascular Surgery, ${ }^{\mathrm{b}}$ Asan Medical Center, University of Ulsan College of Medicine, Seoul, Korea.

Disclosures: Authors have nothing to disclose with regard to commercial support.

Received for publication March 12, 2012; revisions received Nov 15, 2012; accepted for publication Jan 11, 2013; available ahead of print Feb 4, 2013.

Address for reprints: Jong-Min Song, MD, PhD, Asan Medical Center, Division of

Cardiology, University of Ulsan College of Medicine, 388-1 Poongnap2-dong,

Songpa-gu, Seoul 138-736, South Korea (E-mail: jmsong@amc.seoul.kr).

$0022-5223 / \$ 36.00$

Copyright (c) 2014 by The American Association for Thoracic Surgery

http://dx.doi.org/10.1016/j.jtcvs.2013.01.022 pericardiectomy has been associated with a relatively high early mortality/morbidity and low long-term survival, , $^{2-12}$ suggesting that risk stratification before pericardiectomy may be clinically essential. Most studies investigating factors clinically prognostic in patients undergoing pericardiectomy have been conducted in Western countries. ${ }^{2,4,7,8,11,13-16}$ However, preoperative prognostic factors and clinical outcomes after pericardiectomy remain to be clearly elucidated, especially in patients from Asian countries, where the causes of $\mathrm{CP}$ differ from those in Western countries. Furthermore, despite the primary role of Doppler echocardiographic parameters in the diagnosis of $\mathrm{CP}$, their prognostic implications have not been determined. We therefore assessed the clinical and echocardiographic prognostic factors and clinical outcomes after pericardiectomy in patients with CP.

\section{MATERIALS AND METHODS Patient Population}

We enrolled 125 consecutive patients who underwent pericardiectomy for treatment of CP at the Asan Medical Center from January 1, 1996, to September 30, 2010. Diagnosis of CP was based on clinical features, Doppler echocardiography, and other imaging methods.,17,18 Among those patients, 12 with significant aortic or mitral valvular disease, 12 who 


$$
\begin{aligned}
& \text { Abbreviations and Acronyms } \\
& \text { AFB }=\text { acid-fast bacilli } \\
& \text { CP }=\text { constrictive pericarditis } \\
& \text { CT }=\text { computed tomography } \\
& \text { CVP }=\text { central venous pressure } \\
& \text { IVC }=\text { inferior vena cava } \\
& \text { LV }=\text { left ventricular }
\end{aligned}
$$

underwent aortic or mitral valvular surgery, 4 with significant coronary artery stenosis, 7 who underwent percutaneous coronary intervention or coronary artery bypass graft surgery, 3 who underwent cardiac transplantation, 1 with aortic intramural hematoma, and 1 with malignant mesothelioma were excluded for obviating confounding effects of other diseases on patients' prognosis. Patients with coronary artery disease were excluded from the study because all-cause death, the primary clinical end point of this study, might be affected not only by $\mathrm{CP}$ but also by ischemic events in these patients. Patients with significant valvular heart disease or who underwent valve surgery were also excluded because Doppler echocardiographic parameters, such as mitral inflow velocity, might be influenced by these clinical conditions, and mortality may result from valvular complications in such patients. Consequently, a total of 85 patients were included in this study, and the preoperative clinical characteristics of the patients are shown in Table 1. No patients had collagen vascular disease or underwent thoracic irradiation. In addition to these characteristics, we evaluated laboratory findings, echocardiographic and imaging parameters, and surgical methods in these patients.

Of the 36 patients with tuberculous CP, 8 (22.2\%) had chronic granuloma with typical caseous necrosis and positive results in acid-fast bacilli (AFB) staining/culture or polymerase chain reaction for AFB; 20 $(55.6 \%)$ had characteristic radiologic findings suggesting tuberculosis, such as typical tuberculoma, mediastinal lymphadenopathy, or pleuropericarditis; $5(13.9 \%)$ had pleural adenosine deaminase concentrations greater than $40 \mathrm{IU} / \mathrm{L} ; 2(5.6 \%)$ had sputum positive for AFB staining; and $1(2.7 \%)$ showed clinical improvement after treatment with antituberculous drugs.

\section{Echocardiography and Imaging Modalities}

Left ventricular (LV) end-systolic and end-diastolic dimensions were measured using M-mode echocardiography. When M-mode was not suitable for measurement of dimensions because LV long-axis direction was not perpendicular to the echo beam, 2-dimensional echocardiography was used. Because respiratory variation in LV cavity size is a characteristic finding in patients with $\mathrm{CP}, \mathrm{LV}$ dimensions were measured during end-expiration phase, when LV dimensions appeared maximal. LV endsystolic and end-diastolic volumes were measured using the modified Simpson method, and LV ejection fractions were calculated. ${ }^{19} \mathrm{LV}$ volumes were also measured during end-expiration phase. Stroke volume in patients without significant mitral regurgitation was calculated from the difference between end-diastolic and end-systolic volumes. Left atrial dimension was measured at end-systole from the parasternal view. LV and left atrial dimensions and LV volumes were standardized by body surface area of each patient. Early (E) and late (A) diastolic mitral inflow velocities were measured in the early expiratory phase when E velocity was maximal. Pulmonary artery systolic pressure was assessed from the maximal velocity of tricuspid regurgitation jet using a modified Bernoulli's equation. ${ }^{20}$ Right atrial pressure was assumed to be $5 \mathrm{~mm} \mathrm{Hg}$ if the inferior vena cava (IVC) was not dilated $(<1.7 \mathrm{~cm})$ and there was a more than a $50 \%$ decrease in the diameter during inspiration, $10 \mathrm{~mm} \mathrm{Hg}$ if the IVC was dilated with normal inspiratory collapse, and $15 \mathrm{~mm} \mathrm{Hg}$ if the IVC was dilated and did not collapse with inspiration (IVC plethora). ${ }^{19}$
TABLE 1. Preoperative baseline characteristics of 85 patients who underwent pericardiectomy

\begin{tabular}{lc}
\hline Age, $\mathrm{y}$ & $51.8 \pm 13.7$ \\
Male, $\mathrm{n}(\%)$ & $52(61.2)$ \\
Hypertension, $\mathrm{n}(\%)$ & $13(15.3)$ \\
Diabetes mellitus, $\mathrm{n}(\%)$ & $10(11.8)$ \\
Cause, $\mathrm{n}(\%)$ & \\
$\quad$ Idiopathic & $49(57.6)$ \\
$\quad$ Tuberculosis & $36(42.4)$ \\
Symptom duration, mo & $6.2 \pm 7.0$ \\
NYHA III and IV, n (\%) & $30(35.3)$ \\
\hline
\end{tabular}

NYHA, New York Heart Association.

Before pericardiectomy, chest x-rays were taken in all 85 patients, and chest computed tomography (CT) was performed in 76 patients to evaluate pericardial calcification.

\section{Pericardiectomy and Clinical Events}

Complete pericardiectomy was defined as wide excision of the pericardium, anteriorly extending to both phrenic nerves and including the diaphragmatic pericardium. Incomplete pericardiectomy was defined as any pericardial excision that did not meet the criteria for complete pericardiectomy. The effectiveness of pericardiectomy was evaluated by measuring central venous pressures (CVPs) before and 1 day after pericardiectomy, with CVP gap defined as the difference between these values. High CVP was defined as more than $12 \mathrm{~mm} \mathrm{Hg}$. Cardiopulmonary bypass was used in 9 patients $(10.6 \%)$. Early postoperative echocardiography was performed in 81 patients $(95 \%)$ ). Early postoperative constriction was defined as a significant respiratory variation in mitral inflow E-wave velocity $(\geq 25 \%)$ and a prominent increase in diastolic flow reversal with expiration in the hepatic vein on 2-dimensional Doppler echocardiography. ${ }^{17}$ Allcause death was assessed for a median of 38.5 months (range, 0.2-156.9 months)

\section{Statistical Analysis}

Statistical analyses were performed using SPSS 12.0 software (SPSS Inc, Chicago, Ill). Data were expressed as mean \pm standard deviation. Continuous variables were compared using unpaired Student $t$ tests, and the ratios of 2 groups were compared using the Fisher exact test. Univariate and multivariate associations of clinical and echocardiographic variables with all-cause death were assessed using Cox proportional hazards model. Variables with a $P$ value of .10 or less in univariate analyses were candidates

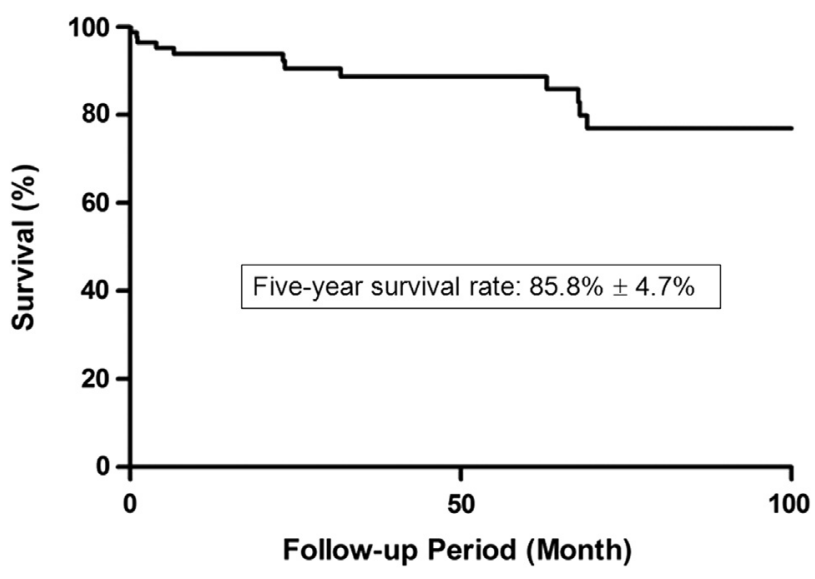

FIGURE 1. Kaplan-Meier curve of survival after pericardiectomy for CP 
TABLE 2. Comparisons of clinical variables between patients who survived and died during follow-up

\begin{tabular}{|c|c|c|c|}
\hline Variables & $\begin{array}{c}\text { Survived } \\
\mathbf{N}=\mathbf{7 0}\end{array}$ & $\begin{array}{c}\text { Died } \\
N=15\end{array}$ & $\boldsymbol{P}$ \\
\hline Age, y & $50.7 \pm 13.3$ & $56.7 \pm 14.9$ & .1 \\
\hline Male, n (\%) & $43(61.4)$ & $9(60.0)$ & 1.00 \\
\hline Hypertension, n (\%) & $10(14.3)$ & $3(20.0)$ & .6 \\
\hline Diabetes mellitus, n (\%) & $6(8.6)$ & $4(26.7)$ & .0 \\
\hline \multicolumn{4}{|l|}{ Cause, n $(\%)$} \\
\hline Idiopathic & $38(54.3)$ & $11(73.3)$ & .2 \\
\hline Tuberculosis & $32(45.7)$ & $4(26.7)$ & .2 \\
\hline Symptom duration, mo & $6.1 \pm 6.7$ & $6.4 \pm 8.4$ & .8 \\
\hline $\begin{array}{l}\text { Preoperative NYHA III } \\
\text { and IV, } \mathrm{n}(\%)\end{array}$ & $23(32.9)$ & $7(46.7)$ & .37 \\
\hline \multicolumn{4}{|l|}{ Physical examinations, $\mathrm{n}(\%)$} \\
\hline JVP elevation & $44(62.9)$ & $10(66.7)$ & 1.00 \\
\hline Pericardial knock & $8(11.4)$ & $1(6.7)$ & 1.00 \\
\hline Peripheral edema & $51(72.9)$ & $10(66.7)$ & .7 \\
\hline Ascites & $15(21.4)$ & $5(33.3)$ & .33 \\
\hline Hepatomegaly & $7(10.0)$ & $2(13.3)$ & 6 \\
\hline \multicolumn{4}{|l|}{ Laboratory findings } \\
\hline Creatinine, $\mathrm{mg} / \mathrm{dL}$ & $1.1 \pm 0.9$ & $1.7 \pm 2.5$ & .1 \\
\hline Albumin, $\mathrm{g} / \mathrm{dL}$ & $3.6 \pm 0.7$ & $3.3 \pm 0.6$ & .10 \\
\hline Aspartate aminotransferase, IU/L & $28.2 \pm 8.8$ & $34.5 \pm 14.7$ & .03 \\
\hline Alanine aminotransferase, IU/L & $20.3 \pm 9.5$ & $25.1 \pm 17.8$ & .13 \\
\hline Alkaline phosphatase, IU/L & $146.3 \pm 95$ & $178.3 \pm 96.4$ & .24 \\
\hline Total bilirubin, $\mathrm{mg} / \mathrm{dL}$ & $1.5 \pm 1.0$ & $1.3 \pm 0.7$ & .34 \\
\hline C-reactive protein, $\mathrm{mg} / \mathrm{dL}$ & $1.6 \pm 3.8$ & $0.9 \pm 0.6$ & .62 \\
\hline Use of diuretics, n (\%) & $51(72.9)$ & $12(80.0)$ & .7 \\
\hline \multicolumn{4}{|l|}{ Electrocardiogram, $\mathrm{n}(\%)$} \\
\hline Atrial fibrillation & $20(28.6)$ & $5(33.3)$ & .75 \\
\hline Atrial flutter & $7(10.0)$ & $0(0.0)$ & .34 \\
\hline Low voltage & $9(12.9)$ & $2(13.3)$ & 1.00 \\
\hline \multicolumn{4}{|l|}{$\mathrm{CT}^{*}$} \\
\hline Pericardial calcification, $\mathrm{n}(\%)$ & $37(57.8)$ & $4(33.3)$ & .20 \\
\hline Pericardial effusion, $\mathrm{n}(\%)$ & $15(23.4)$ & $4(33.3)$ & .48 \\
\hline Pericardial adhesion, $\mathrm{n}(\%)$ & $8(12.5)$ & $2(16.7)$ & .65 \\
\hline Pericardial thickening, $\mathrm{n}(\%)$ & $44(68.8)$ & $9(75.0)$ & 1.00 \\
\hline \multicolumn{4}{|l|}{ Chest x-ray } \\
\hline Pericardial calcification, $\mathrm{n}(\%)$ & $28(40.0)$ & $4(26.7)$ & .5 \\
\hline Cardiothoracic ratio, $\%$ & $52 \pm 6.1$ & $53.2 \pm 5.7$ & .53 \\
\hline \multicolumn{4}{|l|}{ Echocardiography } \\
\hline $\begin{array}{l}\mathrm{LV} \text { end-systolic dimension } \\
\text { index, } \mathrm{mm} / \mathrm{m}^{2}\end{array}$ & $19.0 \pm 3.9$ & $16.6 \pm 2.4$ & .0 \\
\hline $\begin{array}{l}\text { LV end-diastolic dimension } \\
\text { index, } \mathrm{mm} / \mathrm{m}^{2}\end{array}$ & $26.6 \pm 4$ & $24.9 \pm 3.3$ & .1 \\
\hline LA dimension index, $\mathrm{mm} / \mathrm{m}^{2}$ & $28.8 \pm 5.7$ & $29.6 \pm 5.1$ & .62 \\
\hline $\begin{array}{l}\mathrm{LV} \text { end-systolic volume } \\
\text { index, } \mathrm{mL} / \mathrm{m}^{2}\end{array}$ & $13.7 \pm 5.4$ & $11.5 \pm 3.1$ & .20 \\
\hline $\begin{array}{l}\mathrm{LV} \text { end-diastolic volume } \\
\text { index, } \mathrm{mL} / \mathrm{m}^{2}\end{array}$ & $34.4 \pm 10.8$ & $30.8 \pm 8.5$ & .3 \\
\hline Stroke volume index, $\mathrm{mL} / \mathrm{m}^{2}$ & $20.7 \pm 7.1$ & $19.3 \pm 6.3$ & .54 \\
\hline LV ejection fraction, $\%$ & $59.6 \pm 7.5$ & $60.4 \pm 8.3$ & .7 \\
\hline PASP, $\mathrm{mm} \mathrm{Hg}$ & $29.8 \pm 11.3$ & $26.2 \pm 11.7$ & .28 \\
\hline IVC plethora, n $(\%)$ & $68(97.1)$ & $15(100.0)$ & 1.00 \\
\hline E velocity, $\mathrm{cm} / \mathrm{s}$ & $73.4 \pm 20.1$ & $86.8 \pm 26.1$ & .03 \\
\hline A velocity, $\dagger \mathrm{cm} / \mathrm{s}$ & $42.4 \pm 18.1$ & $37.1 \pm 8.9$ & .45 \\
\hline
\end{tabular}

TABLE 2. Continued

\begin{tabular}{lccc}
\hline \multicolumn{1}{c}{ Variables } & $\begin{array}{c}\text { Survived } \\
\mathbf{N}=\mathbf{7 0}\end{array}$ & $\begin{array}{c}\text { Died } \\
\mathbf{N}=\mathbf{1 5}\end{array}$ & $\boldsymbol{P}$ \\
\hline $\mathrm{E} /$ A ratio $\dagger$ & $1.6 \pm 0.7$ & $2.0 \pm 0.5$ & .237 \\
$\mathrm{E}^{\prime}$ velocity, $\ddagger \mathrm{cm} / \mathrm{s}$ & $11.8 \pm 2.9$ & $11.0 \pm 1.2$ & .540 \\
$\mathrm{~A}^{\prime}$ velocity, $\S \mathrm{cm} / \mathrm{s}$ & $8.1 \pm 3.3$ & $8.0 \pm 1.4$ & .984 \\
$\quad$ Maximal pericardial & $9.4 \pm 3.2$ & $11.2 \pm 4$ & .127 \\
$\quad$ thickness, mm & & & \\
Preoperative CVP, mm Hg & $18.8 \pm 5.9$ & $20.5 \pm 6.4$ & .356 \\
Postoperative CVP, mm Hg & $12.9 \pm 3.6$ & $13.0 \pm 5.1$ & .929 \\
CVP gap, mm Hg & $5.8 \pm 5.2$ & $7.5 \pm 6.0$ & .290 \\
High postoperative CVP, n $(\%)$ & $36(51.4)$ & $7(46.7)$ & .782 \\
Cardiopulmonary bypass, $\mathrm{n}(\%)$ & $6(80.6)$ & $3(20.0)$ & .192 \\
Incomplete pericardiectomy, $\mathrm{n}(\%)$ & $14(20.0)$ & $3(20.0)$ & 1.000 \\
Early postoperative constriction, & $32(45.7)$ & $4(26.7)$ & .248
\end{tabular}

$\mathrm{n}(\%)$

NYHA, New York Heart Association; $J V P$, jugular venous pressure; $C T$, computed tomography; $L V$, left ventricular; $L A$, left atrium; $P A S P$, pulmonary artery systolic pressure; $I V C$, inferior vena cava; $C V P$, central venous pressure. * ${ }^{*}$ T was performed in 76 patients. $\dagger$ A velocity and $\mathrm{E} / \mathrm{A}$ ratio were present in 53 patients in normal sinus rhythm. $\ddagger \mathrm{E}^{\prime}$ velocity was available in 43 patients. $\S \mathrm{A}^{\prime}$ velocity was available in 24 patients.

for the multivariate Cox regression analysis. The final models were determined by backward elimination. Schoenfeld residuals and the $\log (-\log$ [survival]) were used to verify that the proportional hazards assumptions were not violated. The optimal cutoff values of continuous variables for predicting mortality were determined using receiver operating characteristic curve analysis. Kaplan-Meier analysis was used to determine the survival, and the difference between groups was analyzed using the log-rank test.

\section{RESULTS \\ Clinical Events}

Of the 85 included patients, $15(17.6 \%)$ died during follow-up after pericardiectomy. The 15 deaths included 1 postoperative in-hospital death and 14 late deaths. Causes of late death were progression of congestive heart failure in 4 patients, sudden cardiac death in 6 patients, infection in 3 patients, and ovarian cancer in 1 patient. The 5-year overall survival was $85.8 \% \pm 4.7 \%$ (Figure 1 ).

Between-group analyses showed that the 15 patients who died during follow-up had higher aspartate aminotransferase, smaller LV end-systolic dimension index, and higher E velocity before pericardiectomy than the 70 patients who survived (Table 2, Figure 2). Of the 85 patients, complete pericardiectomy was possible in 68 patients $(80.0 \%)$, but there was no difference in the proportions who underwent complete pericardiectomy between patients who survived and died during follow-up. There was no significant between-group difference in early postoperative constriction. Among 36 patients who showed early postoperative constriction in Doppler echocardiography, $28(77.8 \%)$ presented an uneventful stable clinical condition without reoperation, $4(11.1 \%)$ were admitted because of heart failure, $1(2.8 \%)$ was admitted because of coronary artery disease, and $3(8.3 \%)$ eventually died during follow-up. 

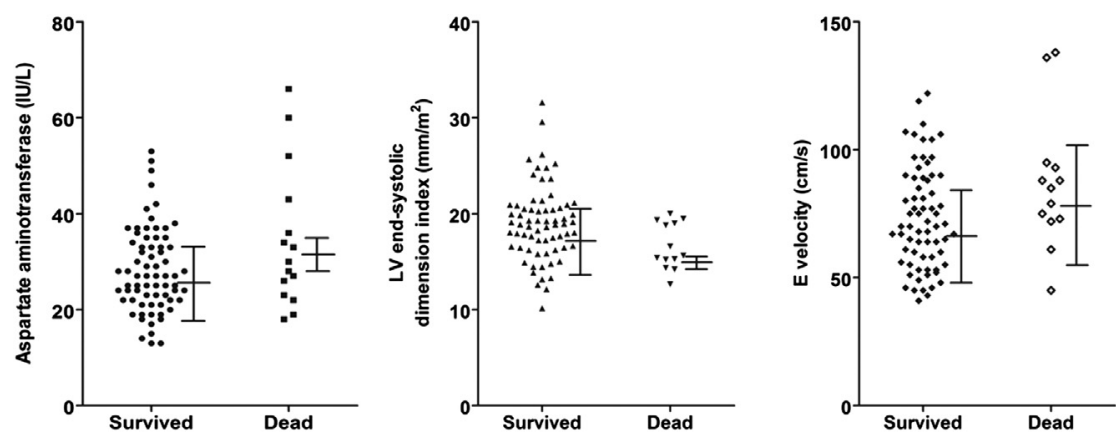

FIGURE 2. Comparisons of aspartate aminotransferase, LV end-systolic dimension index, and E velocity between patients who survived and died during follow-up after pericardiectomy. $L V$, Left ventricular.

\section{Predictors of Clinical Events}

Univariate Cox regression analysis showed that older age, high aspartate aminotransferase, small LV end-systolic dimension index, and high $\mathrm{E}$ velocity were significant predictors of mortality, with the presence of diabetes mellitus, high alanine aminotransferase, and use of cardiopulmonary bypass during pericardiectomy showing marginal significance $(P<.1)$ (Table 3$)$. Multivariate Cox proportional hazard analysis including these variables showed that diabetes mellitus and high $\mathrm{E}$ velocity before pericardiectomy were independent predictors of mortality after pericardiectomy. Receiver operating characteristic curve analyses showed that the best preoperative cutoff value for $E$ velocity in predicting all-cause death after pericardiectomy was $71 \mathrm{~cm} / \mathrm{s}$ (sensitivity of $84.6 \%$ and specificity of $52.2 \%$; Figure 3 ). There was a significant difference in survival between groups divided by this cutoff value of $\mathrm{E}$ velocity $(P=.029$; Figure 4). Five-year survivals of patients with high and low preoperative E velocity were $83.6 \% \pm 5.8 \%$ and $95.2 \% \pm 4.6 \%$, respectively. The survival of patients with diabetes mellitus tended to be lower than that of patients without diabetes mellitus, but there was no statistical significance $(P=.081)$. The 47 patients with high E velocity $(>71 \mathrm{~cm} / \mathrm{s})$ before pericardiectomy were more likely to have atrial fibrillation than 38 patients with low E velocity (Table 4). The former tended to show pericardial calcification and thickening on chest $\mathrm{x}$-ray and CT before surgery and a large left atrial dimension index with marginal significance. There was no significant difference in early postoperative constriction between the 2 groups. The patients with high preoperative $E$ velocity showed significantly larger left atrial dimension index, higher pulmonary artery systolic pressure, and higher E velocity in early postoperative echocardiography than those with low preoperative E velocity. There was a significant positive correlation between preoperative and early postoperative E velocities $(r=0.480$, $P<.001$; Figure 5). Among the 70 patients who survived, $3(8.8 \%)$ and $2(5.6 \%)$ with high and low preoperative $\mathrm{E}$ velocity were admitted to the hospital for heart failure during follow-up, respectively $(P=.669)$.

\section{DISCUSSION \\ Clinical Events}

We found that the postoperative in-hospital mortality rate after pericardiectomy was $1.2 \%$, somewhat low when compared with previous reports, in which perioperative

TABLE 3. Univariate and multivariate Cox proportional hazard model for predicting all-cause death

\begin{tabular}{|c|c|c|c|c|c|}
\hline \multirow[b]{2}{*}{ Variables } & \multicolumn{2}{|c|}{ Univariate } & \multicolumn{3}{|c|}{ Multivariate } \\
\hline & HR & $P$ & HR & $95 \%$ CI & $P$ \\
\hline Age, y & 1.039 & .046 & - & - & - \\
\hline Male & 0.830 & .726 & - & - & - \\
\hline Hypertension & 0.980 & .976 & - & - & - \\
\hline Diabetes mellitus & 2.691 & .093 & 4.610 & $1.226-17.337$ & .024 \\
\hline \multicolumn{6}{|l|}{ Cause } \\
\hline Idiopathic & 1.815 & .310 & - & - & - \\
\hline Tuberculosis & 0.551 & .310 & - & - & - \\
\hline Symptom duration, mo & 0.980 & .552 & - & - & - \\
\hline $\begin{array}{l}\text { Preoperative NYHA III and } \\
\text { IV }\end{array}$ & 1.805 & .255 & - & - & - \\
\hline \multicolumn{6}{|l|}{ Physical examinations } \\
\hline JVP elevation & 1.109 & .850 & - & - & - \\
\hline Pericardial knock & 0.525 & .536 & - & - & - \\
\hline Peripheral edema & 0.648 & .431 & - & - & - \\
\hline Ascites & 0.964 & .950 & - & - & - \\
\hline Hepatomegaly & 0.756 & .725 & - & - & - \\
\hline \multicolumn{6}{|l|}{ Laboratory findings } \\
\hline Creatinine, $\mathrm{mg} / \mathrm{dL}$ & 1.231 & .180 & - & - & - \\
\hline Albumin, $\mathrm{g} / \mathrm{dL}$ & 0.575 & .117 & - & - & - \\
\hline $\begin{array}{l}\text { Aspartate } \\
\text { aminotransferase, IU/L }\end{array}$ & 1.061 & .008 & - & - & - \\
\hline $\begin{array}{l}\text { Alanine aminotransferase, } \\
\text { IU/L }\end{array}$ & 1.039 & .052 & - & - & - \\
\hline Alkaline phosphatase, IU/L & 1.001 & .658 & - & - & - \\
\hline Total bilirubin, $\mathrm{mg} / \mathrm{dL}$ & 0.955 & .881 & - & - & - \\
\hline $\mathrm{C}$-reactive protein, $\mathrm{mg} / \mathrm{dL}$ & 0.862 & .570 & - & - & - \\
\hline Use of diuretics & 1.463 & .556 & - & - & - \\
\hline \multicolumn{6}{|l|}{ Electrocardiogram } \\
\hline Atrial fibrillation & 1.384 & .554 & - & - & - \\
\hline Atrial flutter & 0.043 & .454 & - & - & - \\
\hline Low voltage & 1.002 & .998 & - & - & - \\
\hline
\end{tabular}


TABLE 3. Continued

\begin{tabular}{|c|c|c|c|c|c|}
\hline \multirow[b]{2}{*}{ Variables } & \multicolumn{2}{|c|}{ Univariate } & \multicolumn{3}{|c|}{ Multivariate } \\
\hline & HR & $\boldsymbol{P}$ & HR & $95 \% \mathrm{CI}$ & $P$ \\
\hline \multicolumn{6}{|l|}{ CT } \\
\hline Pericardial calcification & 0.456 & .204 & - & - & - \\
\hline Pericardial effusion & 2.095 & .241 & - & - & - \\
\hline Pericardial adhesion & 2.930 & .193 & - & - & - \\
\hline Pericardial thickening & 1.454 & .577 & - & - & - \\
\hline \multicolumn{6}{|l|}{ Chest x-ray } \\
\hline Pericardial calcification & 0.559 & .381 & - & - & - \\
\hline Cardiothoracic ratio, $\%$ & 1.034 & .505 & - & - & - \\
\hline \multicolumn{6}{|l|}{ Echocardiography } \\
\hline $\begin{array}{l}\text { LV end-systolic dimension } \\
\text { index, } \mathrm{mm} / \mathrm{m}^{2}\end{array}$ & 0.821 & .038 & 0.815 & $0.643-1.031$ & .08 \\
\hline $\begin{array}{l}\text { LV end-diastolic dimension } \\
\text { index, } \mathrm{mm} / \mathrm{m}^{2}\end{array}$ & 0.907 & .250 & - & - & - \\
\hline $\begin{array}{l}\text { LA dimension index, } \\
\mathrm{mm} / \mathrm{m}^{2}\end{array}$ & 1.002 & .974 & - & - & - \\
\hline $\begin{array}{l}\text { LV end-systolic volume } \\
\text { index, } \mathrm{mL} / \mathrm{m}^{2}\end{array}$ & 0.948 & .442 & - & - & - \\
\hline $\begin{array}{l}\mathrm{LV} \text { end-diastolic volume } \\
\text { index, } \mathrm{mL} / \mathrm{m}^{2}\end{array}$ & 0.97 & .300 & - & 一 & - \\
\hline Stroke volume, $\mathrm{mL} / \mathrm{m}^{2}$ & 0.953 & .299 & - & - & - \\
\hline LV ejection fraction, $\%$ & 0.994 & .856 & - & - & - \\
\hline PASP, mm Hg & 0.99 & .702 & - & - & - \\
\hline IVC plethora & 22.355 & .563 & - & - & - \\
\hline E velocity, $\mathrm{cm} / \mathrm{s}$ & 1.034 & .011 & 1.050 & $1.018-1.083$ & .002 \\
\hline A velocity, $\mathrm{cm} / \mathrm{s}$ & 0.991 & .717 & - & - & - \\
\hline E/A ratio & 1.340 & .546 & - & - & - \\
\hline $\mathrm{E}^{\prime}$ velocity, $\mathrm{cm} / \mathrm{s}$ & 1.000 & .999 & - & - & - \\
\hline $\mathrm{A}^{\prime}$ velocity, $\mathrm{cm} / \mathrm{s}$ & 1.293 & .486 & - & - & - \\
\hline $\begin{array}{l}\text { Maximal pericardial } \\
\text { thickness, } \mathrm{mm}\end{array}$ & 1.156 & .114 & - & - & - \\
\hline Preoperative CVP, $\mathrm{mm} \mathrm{Hg}$ & 1.022 & .605 & - & - & - \\
\hline Postoperative CVP, $\mathrm{mm} \mathrm{Hg}$ & 1.031 & .638 & - & - & - \\
\hline CVP gap, mm Hg & 1.022 & .658 & - & - & - \\
\hline High postoperative CVP & 1.02 & .970 & - & - & - \\
\hline Cardiopulmonary bypass & 3.528 & .060 & - & - & - \\
\hline Incomplete pericardiectomy & 1.708 & .426 & - & - & - \\
\hline $\begin{array}{l}\text { Early postoperative } \\
\text { constriction }\end{array}$ & 0.924 & .899 & - & - & - \\
\hline
\end{tabular}

HR, Hazard ratio; $C I$, confidence interval; NYHA, New York Heart Association; $J V P$, jugular venous pressure; $C T$, computed tomography; $L V$, left ventricular; $L A$, left atrium; $P A S P$, pulmonary artery systolic pressure; $I V C$, inferior vena cava; $C V P$, central venous pressure.

mortality rates after pericardiectomy ranged from $2.3 \%$ to $12 \% .^{2-9}$ The relatively good postoperative prognosis we observed may be due to our patient population, in which the most common cause of $\mathrm{CP}$ was idiopathic. Patients with idiopathic $\mathrm{CP}$ were reported to have the best prognosis after pericardiectomy. ${ }^{6,8}$ Furthermore, we excluded patients with significant valvular and coronary artery disease, and other serious comorbidities to obviate confounding effects of other diseases on patients' mortality and evaluate the pure prognosis of patients with isolated $\mathrm{CP}$ after pericardiectomy. Although

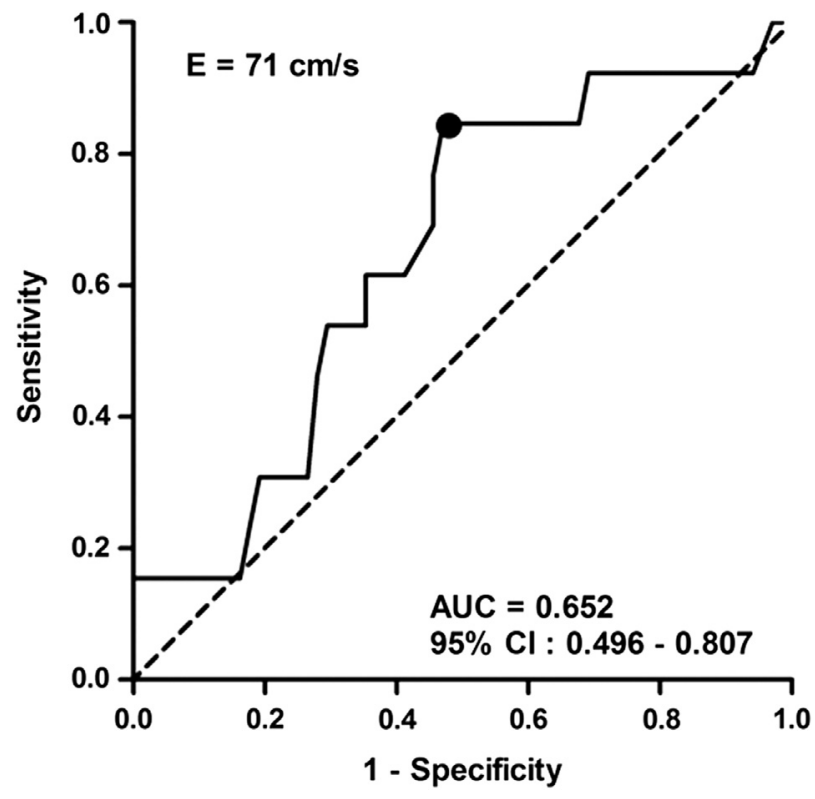

FIGURE 3. Receiver operating characteristic curve of transmitral $E$ velocity (E). Dot on the curve denotes best cutoff value. AUC, Area under curve; $C I$, confidence interval.

mortality rates after pericardiectomy in patients with postradiotherapy $\mathrm{CP}$ have been reported to be high, ${ }^{2,6,8}$ none of our patients had this cause. Most patients with postirradiation $\mathrm{CP}$ underwent radiotherapy to treat lymphoma and breast cancer. Patients with Hodgkin's lymphoma are frequently administered radiotherapy for mediastinal lymph nodes. However, this disease is more prevalent in the United States and Europe than in Eastern countries, including Korea. The most common subtype of malignant lymphoma in Korea is diffuse large B-cell lymphoma, ${ }^{21}$ which is usually treated with chemotherapy and rarely with radiotherapy. In addition, $36 \%$ of our patients had tuberculous $\mathrm{CP}$, which was found to be a major cause in only a few reports. ${ }^{3,9}$ We found that the 5-year overall survival after pericardiectomy $(85.8 \%)$ tended to be higher than in previous reports, in which survival ranged from $64 \%$ to $85 \%{ }^{4,5,7,9,11,16}$

\section{Predictors of Clinical Events}

We found that diabetes mellitus and high E velocity before pericardiectomy were independent predictors of clinical events after pericardiectomy. A novel finding of our study was that a Doppler echocardiographic parameter, early expiratory transmitral $\mathrm{E}$ velocity, which is important in $\mathrm{CP}$ diagnosis, was also prognostic in these patients. The explanation of mechanism that high $E$ velocity was associated with mortality after pericardiectomy would be speculative. However, this Doppler parameter may represent the severity of $\mathrm{CP}$, and a high transmitral $\mathrm{E}$ velocity may reflect a high left atrial pressure resulting from severe 

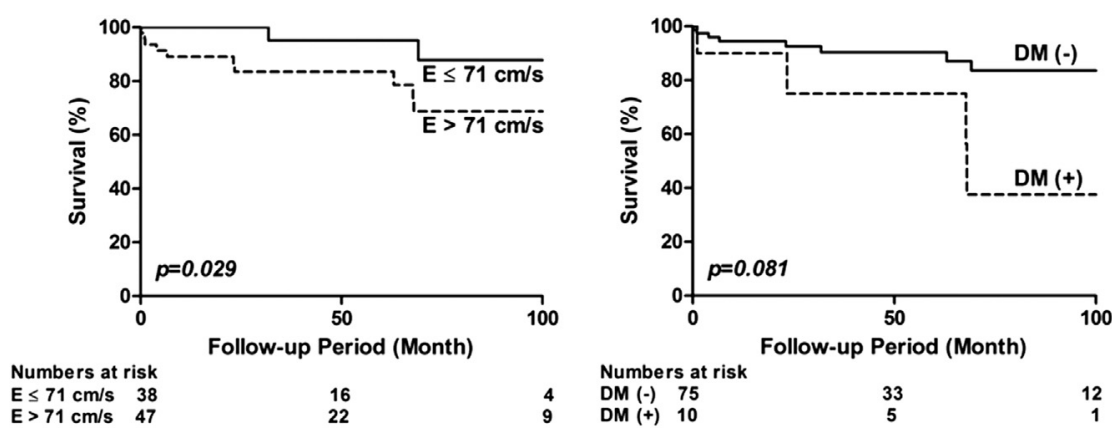

FIGURE 4. Kaplan-Meier curves showing survival after pericardiectomy for patients with CP relative to transmitral E velocity (E) and DM. DM, Diabetes mellitus.

pericardial constriction. In this study, a high E velocity was significantly associated with atrial fibrillation and marginally associated with pericardial thickening and calcification, which suggested that high $\mathrm{E}$ velocity in patients with $\mathrm{CP}$ is

TABLE 4. Comparisons of clinical variables between patients who showed preoperative high and low $E$ velocity

\begin{tabular}{|c|c|c|c|}
\hline Variables & $\begin{array}{c}\text { Low E } \\
\text { velocity } \\
\mathbf{N}=\mathbf{3 8} \\
\end{array}$ & $\begin{array}{l}\text { High } E \\
\text { velocity } \\
N=47 \\
\end{array}$ & $\boldsymbol{P}$ \\
\hline Age, y & $49.7 \pm 12.2$ & $53.5 \pm 14.7$ & .196 \\
\hline Male, n (\%) & $24(63.2)$ & $28(59.6)$ & .824 \\
\hline Hypertension, n (\%) & $6(15.8)$ & 7 (14.9) & 1.000 \\
\hline Diabetes mellitus, n (\%) & $4(10.5)$ & $6(12.8)$ & 1.000 \\
\hline \multicolumn{4}{|l|}{ Cause, n (\%) } \\
\hline Idiopathic & $19(50.0)$ & $30(63.8)$ & .270 \\
\hline Tuberculosis & $19(50.0)$ & $17(36.2)$ & .270 \\
\hline Symptom duration, mo & $6.4 \pm 5.8$ & $5.9 \pm 7.9$ & .753 \\
\hline $\begin{array}{l}\text { Preoperative NYHA III } \\
\text { and IV, } \mathrm{n}(\%)\end{array}$ & $12(31.6)$ & $18(38.3)$ & .649 \\
\hline \multicolumn{4}{|l|}{ Physical examinations, n (\%) } \\
\hline JVP elevation & $23(60.5)$ & $31(66.0)$ & .655 \\
\hline Pericardial knock & $3(7.9)$ & $6(12.8)$ & .725 \\
\hline Peripheral edema & $28(73.7)$ & $33(70.2)$ & .811 \\
\hline Ascites & $8(21.1)$ & $12(25.5)$ & .798 \\
\hline Hepatomegaly & $3(7.9)$ & $6(12.8)$ & .725 \\
\hline \multicolumn{4}{|l|}{ Laboratory findings } \\
\hline Creatinine, mg/dL & $1.2 \pm 1.1$ & $1.2 \pm 1.5$ & .922 \\
\hline Albumin, g/dL & $3.6 \pm 0.6$ & $3.5 \pm 0.7$ & .511 \\
\hline $\begin{array}{l}\text { Aspartate aminotransferase, } \\
\text { IU/L }\end{array}$ & $27.9 \pm 9.6$ & $30.5 \pm 10.7$ & .244 \\
\hline $\begin{array}{l}\text { Alanine aminotransferase, } \\
\text { IU/L }\end{array}$ & $21.6 \pm 11.1$ & $20.8 \pm 11.8$ & .739 \\
\hline Alkaline phosphatase, IU/L & $144.7 \pm 94.2$ & $157.8 \pm 97.0$ & .531 \\
\hline Total bilirubin, mg/dL & $1.4 \pm 0.9$ & $1.5 \pm 1.0$ & .696 \\
\hline C-reactive protein, $\mathrm{mg} / \mathrm{dL}$ & $1.3 \pm 3.7$ & $1.8 \pm 3.5$ & .615 \\
\hline Use of diuretics, n (\%) & $28(73.7)$ & $35(74.5)$ & 1.000 \\
\hline \multicolumn{4}{|l|}{ Electrocardiogram, n (\%) } \\
\hline Atrial fibrillation & $2(5.3)$ & $23(48.9)$ & $<.001$ \\
\hline Atrial flutter & $3(7.9)$ & $4(8.5)$ & 1.000 \\
\hline Low voltage & $4(10.5)$ & $7(14.9)$ & .747 \\
\hline
\end{tabular}

associated with disease severity. In patients with severe $\mathrm{CP}$, myocardial involvement of pericardial inflammation, dense adhesion of pericardium with myocardium, and severe calcification of the epicardium that could not be completely

TABLE 4. Continued

\begin{tabular}{|c|c|c|c|}
\hline Variables & $\begin{array}{c}\text { Low } \mathbf{E} \\
\text { velocity } \\
\mathbf{N}=\mathbf{3 8}\end{array}$ & $\begin{array}{l}\text { High } E \\
\text { velocity } \\
N=47\end{array}$ & $\boldsymbol{P}$ \\
\hline \multicolumn{4}{|l|}{$\mathrm{CT}^{*}$} \\
\hline Pericardial calcification, n $(\%)$ & $14(41.2)$ & $27(64.3)$ & .064 \\
\hline Pericardial effusion, $\mathrm{n}(\%)$ & $9(26.5)$ & $10(23.8)$ & .797 \\
\hline Pericardial adhesion, n (\%) & $3(8.8)$ & $7(16.7)$ & .497 \\
\hline Pericardial thickening, $\mathrm{n}(\%)$ & $20(58.8)$ & 33 (78.6) & .081 \\
\hline \multicolumn{4}{|l|}{ Chest x-ray } \\
\hline Pericardial calcification, $\mathrm{n}(\%)$ & $10(26.3)$ & $22(46.8)$ & .070 \\
\hline Cardiothoracic ratio, $\%$ & $51.3 \pm 6.4$ & $52.9 \pm 5.7$ & .245 \\
\hline \multicolumn{4}{|l|}{ Echocardiography } \\
\hline $\begin{array}{l}\text { LV end-systolic dimension } \\
\text { index, } \mathrm{mm} / \mathrm{m}^{2}\end{array}$ & $18.7 \pm 3.9$ & $18.5 \pm 3.8$ & .831 \\
\hline $\begin{array}{l}\text { LV end-diastolic dimension } \\
\text { index, } \mathrm{mm} / \mathrm{m}^{2}\end{array}$ & $26.5 \pm 4.2$ & $26.1 \pm 3.7$ & .691 \\
\hline LA dimension index, $\mathrm{mm} / \mathrm{m}^{2}$ & $27.6 \pm 5.2$ & $30 \pm 5.6$ & .058 \\
\hline $\begin{array}{l}\mathrm{LV} \text { end-systolic volume index, } \\
\mathrm{mL} / \mathrm{m}^{2}\end{array}$ & $12.7 \pm 5$ & $13.9 \pm 5.3$ & .305 \\
\hline $\begin{array}{l}\text { LV end-diastolic volume index, } \\
\mathrm{mL} / \mathrm{m}^{2}\end{array}$ & $33 \pm 11.3$ & $34.6 \pm 9.8$ & .504 \\
\hline Stroke volume index, $\mathrm{mL} / \mathrm{m}^{2}$ & $20.3 \pm 7.9$ & $20.7 \pm 6.1$ & .804 \\
\hline LV ejection fraction, $\%$ & $59.5 \pm 7.7$ & $59.9 \pm 7.5$ & .818 \\
\hline PASP, mm Hg & $27.3 \pm 9.2$ & $30.7 \pm 12.7$ & .183 \\
\hline IVC plethora, n (\%) & $37(97.4)$ & $46(97.9)$ & 1.000 \\
\hline E velocity, $\mathrm{cm} / \mathrm{s}$ & $57.5 \pm 9.0$ & $91.4 \pm 16.2$ & $<.001$ \\
\hline A velocity, $\dagger \mathrm{cm} / \mathrm{s}$ & $41.2 \pm 18.2$ & $42.6 \pm 15.9$ & .769 \\
\hline $\mathrm{E} / \mathrm{A}$ ratio $\dagger$ & $1.4 \pm 0.5$ & $2.1 \pm 0.7$ & $<.001$ \\
\hline $\mathrm{E}^{\prime}$ velocity, $\ddagger \mathrm{cm} / \mathrm{s}$ & $11.6 \pm 3.0$ & $11.8 \pm 2.7$ & .861 \\
\hline $\mathrm{A}^{\prime}$ velocity, $\oint \mathrm{cm} / \mathrm{s}$ & $7.7 \pm 3.4$ & $8.5 \pm 3.0$ & .566 \\
\hline $\begin{array}{l}\text { Maximal pericardial } \\
\text { thickness, } \mathrm{mm}\end{array}$ & $9.0 \pm 3.1$ & $10.4 \pm 3.5$ & .123 \\
\hline Preoperative CVP, mm Hg & $18.5 \pm 5.8$ & $19.5 \pm 6.2$ & .422 \\
\hline Postoperative CVP, mm Hg & $12.2 \pm 4.4$ & $13.5 \pm 3.4$ & .120 \\
\hline CVP gap, mm Hg & $6.3 \pm 5.4$ & $5.9 \pm 5.3$ & .764 \\
\hline
\end{tabular}


TABLE 4. Continued

\begin{tabular}{|c|c|c|c|}
\hline Variables & $\begin{array}{c}\text { Low } \mathbf{E} \\
\text { velocity } \\
\mathbf{N}=\mathbf{3 8} \\
\end{array}$ & $\begin{array}{c}\text { High } E \\
\text { velocity } \\
N=47 \\
\end{array}$ & $\boldsymbol{P}$ \\
\hline High postoperative CVP, n (\%) & $16(42.1)$ & $27(57.4)$ & .193 \\
\hline Cardiopulmonary bypass, $\mathrm{n}(\%)$ & $2(5.3)$ & $7(14.9)$ & .179 \\
\hline $\begin{array}{l}\text { Incomplete pericardiectomy, } \\
\mathrm{n}(\%)\end{array}$ & $6(15.8)$ & $11(23.4)$ & .426 \\
\hline $\begin{array}{l}\text { Early postoperative constriction, } \\
\qquad \# \mathrm{n}(\%)\end{array}$ & $13(35.1)$ & $23(51.1)$ & .182 \\
\hline \multicolumn{4}{|l|}{ Early postoperative echocardiography\# } \\
\hline $\begin{array}{l}\text { LV end-systolic dimension } \\
\text { index, } \mathrm{mm} / \mathrm{m}^{2}\end{array}$ & $17.3 \pm 3.4$ & $17.2 \pm 3.6$ & .881 \\
\hline $\begin{array}{l}\text { LV end-diastolic dimension } \\
\text { index }, \mathrm{mm} / \mathrm{m}^{2}\end{array}$ & $25.6 \pm 4.0$ & $25.6 \pm 3.9$ & .996 \\
\hline LA dimension index, $\mathrm{mm} / \mathrm{m}^{2}$ & $25.7 \pm 6.0$ & $29.6 \pm 4.8$ & .002 \\
\hline $\begin{array}{l}\mathrm{LV} \text { end-systolic volume index, } \\
\mathrm{mL} / \mathrm{m}^{2}\end{array}$ & $14.1 \pm 6.2$ & $14.9 \pm 8.1$ & 639 \\
\hline $\begin{array}{l}\text { LV end-diastolic volume index, } \\
\mathrm{mL} / \mathrm{m}^{2}\end{array}$ & $35.4 \pm 10.2$ & $36.8 \pm 11.8$ & .579 \\
\hline Stroke volume index, $\mathrm{mL} / \mathrm{m}^{2}$ & $21.3 \pm 6.2$ & $21.9 \pm 6.7$ & 669 \\
\hline LV ejection fraction, $\%$ & $61.0 \pm 8.1$ & $60.7 \pm 9.6$ & .892 \\
\hline PASP, $\mathrm{mm} \mathrm{Hg}$ & $24.5 \pm 11.9$ & $31.7 \pm 17.5$ & .049 \\
\hline IVC plethora, $\mathrm{n}(\%)$ & $16(47.1)$ & $28(65.1)$ & .164 \\
\hline E velocity, $\mathrm{cm} / \mathrm{s}$ & $65.6 \pm 14.2$ & $83.7 \pm 21.2$ & $<.001$ \\
\hline A velocity, $* * \mathrm{~cm} / \mathrm{s}$ & $44.8 \pm 13.9$ & $50.8 \pm 33.1$ & .351 \\
\hline E/A ratio** & $1.6 \pm 0.6$ & $1.9 \pm 0.9$ & .121 \\
\hline $\mathrm{E}^{\prime}$ velocity, $\dagger \dagger \mathrm{cm} / \mathrm{s}$ & $8.7 \pm 2.2$ & $9.9 \pm 2.3$ & .175 \\
\hline $\mathrm{A}^{\prime}$ velocity, 拉 $\mathrm{cm} / \mathrm{s}$ & $8.3 \pm 2.5$ & $7.3 \pm 2.9$ & .451 \\
\hline
\end{tabular}

NYHA, New York Heart Association; $J V P$, jugular venous pressure; $C T$, computed tomography; $L V$, left ventricular; $L A$, left atrium; $P A S P$, pulmonary artery systolic pressure; $I V C$, inferior vena cava; $C V P$, central venous pressure. ${ }^{*} \mathrm{CT}$ was performed in 76 patients. $\dagger \mathrm{A}$ velocity and $\mathrm{E} / \mathrm{A}$ ratio were present in 53 patients in normal sinus rhythm. $\ddagger \mathrm{E}^{\prime}$ velocity was available in 43 patients. $\S \mathrm{A}^{\prime}$ velocity was available in 24 patients. \#Early postoperative echocardiography was performed in 81 patients. **Postoperative A velocity and E/A ratio were present in 56 patients in normal sinus

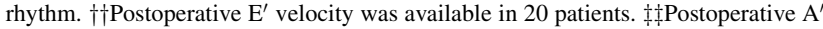
velocity was available in 19 patients.

removed by pericardiectomy might contribute to an increase in E velocity. ${ }^{22}$ The possibility of myocardial involvement of pericardial inflammation was supported by our findings that there was a significant positive correlation between preoperative and early postoperative $\mathrm{E}$ velocities, and early postoperative left atrial dimension index, E velocity, and pulmonary artery systolic pressure were higher in patients with high preoperative E velocity than in those with low velocity, even though there was no significant difference in early postoperative constriction between the 2 groups. It was reported that diastolic filling characteristics remain abnormal in a substantial number of patients with $\mathrm{CP}$ after pericardiectomy, and these abnormalities may resolve gradually but can persist. ${ }^{23}$

High preoperative transmitral $\mathrm{E}$ velocity also might be caused by combined myocardial restriction, which could be found in secondary $\mathrm{CP}$, and this might explain a persistent high E velocity after pericardiectomy. However, this hypothesis is not solidly supported by our data, because there

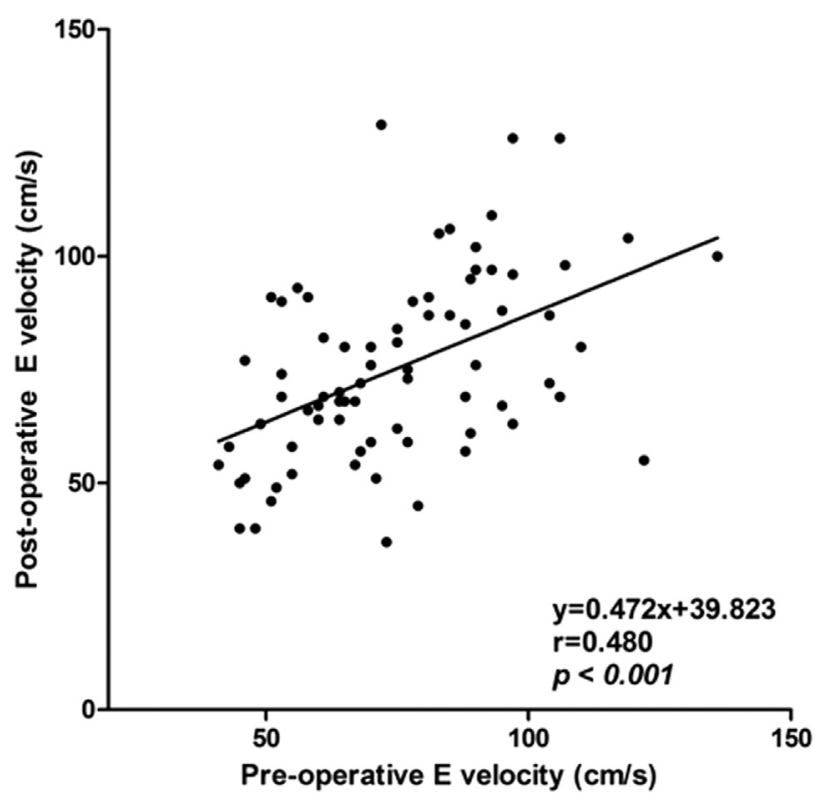

FIGURE 5. Correlation between preoperative and postoperative $\mathrm{E}$ velocities.

was no postirradiation $\mathrm{CP}$ in our study population and patients with significant valvular and ischemic heart diseases were excluded. Furthermore, the average value of $\mathrm{E}^{\prime}$ velocity was high before pericardiectomy and remained relatively high even after pericardiectomy, suggestive of a low probability of myocardial restriction. ${ }^{24}$

The clinical cutoff value for preoperative E velocity we have reported may help identify patients with $\mathrm{CP}$ at high risk after pericardiectomy. Because patients with $\mathrm{CP}$ do not have any other therapeutic options, pericardiectomy cannot be refused even to patients at high risk. However, the findings derived from this study can help to stratify risks and prepare more intensive surgical procedure and postoperative care after pericardiectomy for the patients at high risk.

\section{Study Limitations}

No invasive hemodynamic measurements were routinely implemented; thus, known invasive prognostic parameters, such as right ventricular end-diastolic pressure and right atrial pressure, were not directly measured. ${ }^{4,11}$ However, we found that CVP measured before pericardiectomy and plethora of IVC, a noninvasive indicator of right atrial pressure, were not independent predictors of mortality. Finally, our results may be mainly applied to patients with $\mathrm{CP}$ caused by idiopathic and tuberculous pericarditis, and may not be extrapolated to patients with $\mathrm{CP}$ due to other causes, such as postirradiation, postsurgical, and collagen vascular disease. The prognosis of patients with postsurgical CP may be associated with the severity of underlying valvular or ischemic heart diseases. The cutoff value of $\mathrm{E}$ velocity determined in this study cannot be 
applied to patients with significant mitral valvular disease and patients with a prosthetic mitral valve for predicting prognosis after pericardiectomy.

\section{CONCLUSIONS}

Preoperative high E velocity and diabetes mellitus were predictors of poor prognosis after pericardiectomy in patients with chronic CP. These results suggest that preoperative Doppler echocardiographic evaluation may be valuable not only for diagnosing $\mathrm{CP}$ but also for predicting prognosis after pericardiectomy.

\section{References}

1. Myers RB, Spodick DH. Constrictive pericarditis: clinical and pathophysiologic characteristics. Am Heart J. 1999;138:219-32.

2. Ling LH, Oh JK, Schaff HV, Danielson GK, Mahoney DW, Seward JB, et al. Constrictive pericarditis in the modern era: evolving clinical spectrum and impact on outcome after pericardiectomy. Circulation. 1999;100:1380-6.

3. Nataf P, Cacoub P, Dorent R, Jault F, Bors V, Pavie A, et al. Results of subtotal pericardiectomy for constrictive pericarditis. Eur J Cardiothorac Surg. 1993;7: 252-6.

4. Seifert FC, Miller DC, Oesterle SN, Oyer PE, Stinson EB, Shumway NE. Surgical treatment of constrictive pericarditis: analysis of outcome and diagnostic error. Circulation. 1985;72:II264-73.

5. Aagaard MT, Haraldsted VY. Chronic constrictive pericarditis treated with total pericardiectomy. Thorac Cardiovasc Surg. 1984;32:311-4.

6. Cameron J, Oesterle SN, Baldwin JC, Hancock EW. The etiologic spectrum of constrictive pericarditis. Am Heart J. 1987;113:354-60.

7. DeValeria PA, Baumgartner WA, Casale AS, Greene PS, Cameron DE, Gardner TJ, et al. Current indications, risks, and outcome after pericardiectomy. Ann Thorac Surg. 1991;52:219-24.

8. Bertog SC, Thambidorai SK, Parakh K, Schoenhagen P, Ozduran V, Houghtaling PL, et al. Constrictive pericarditis: etiology and cause-specific survival after pericardiectomy. J Am Coll Cardiol. 2004;43:1445-52.

9. Arsan S, Mercan S, Sarigul A, Atasoy S, Demircin M, Dogan R, et al. Long-term experience with pericardiectomy: analysis of 105 consecutive patients. Thorac Cardiovasc Surg. 1994;42:340-4.

10. Hehrlein FW, Moosdorf R, Pitton M, Dapper F. The role of pericardiectomy in pericardial disorders. Eur Heart J. 1991;12(Suppl D):7-9.

11. Tirilomis T, Unverdorben S, von der Emde J. Pericardectomy for chronic constrictive pericarditis: risks and outcome. Eur J Cardiothorac Surg. 1994;8: 487-92.
12. Gopaldas RR, Dao TK, Caron NR, Markley JG. Predictors of in-hospital complications after pericardiectomy: a nationwide outcomes study. J Thorac Cardiovasc Surg. 2012 May 9. [Epub ahead of print]

13. Ling LH, Oh JK, Breen JF, Schaff HV, Danielson GK, Mahoney DW, et al. Calcific constrictive pericarditis: is it still with us? Ann Intern Med. 2000;132: 444-50.

14. Ha JW, Oh JK, Schaff HV, Ling LH, Higano ST, Mahoney DW, et al. Impact of left ventricular function on immediate and long-term outcomes after pericardiectomy in constrictive pericarditis. J Thorac Cardiovasc Surg. 2008;136:1136-41.

15. Chowdhury UK, Subramaniam GK, Kumar AS, Airan B, Singh R, Talwar S, et al. Pericardiectomy for constrictive pericarditis: a clinical, echocardiographic, and hemodynamic evaluation of two surgical techniques. Ann Thorac Surg. 2006 81:522-9.

16. Bozbuga N, Erentug V, Eren E, Erdogan HB, Kirali K, Antal A, et al. Pericardiectomy for chronic constrictive tuberculous pericarditis: risks and predictors of survival. Tex Heart Inst J. 2003;30:180-5.

17. Oh JK, Hatle LK, Seward JB, Danielson GK, Schaff HV, Reeder GS, et al. Diagnostic role of doppler echocardiography in constrictive pericarditis. $J$ Am Coll Cardiol. 1994;23:154-62.

18. Dal-Bianco JP, Sengupta PP, Mookadam F, Chandrasekaran K, Tajik AJ, Khandheria BK. Role of echocardiography in the diagnosis of constrictive pericarditis. J Am Soc Echocardiogr. 2009;22:24-33; quiz 103-4.

19. Lang RM, Bierig M, Devereux RB, Flachskampf FA, Foster E, Pellikka PA, et al. Recommendations for chamber quantification: a report from the American Society of Echocardiography's Guidelines and Standards Committee and the Chamber Quantification Writing Group, developed in conjunction with the European Association of Echocardiography, a branch of the European Society of Cardiology. J Am Soc Echocardiogr. 2005;18:1440-63.

20. Yock PG, Popp RL. Noninvasive estimation of right ventricular systolic pressure by doppler ultrasound in patients with tricuspid regurgitation. Circulation. 1984; 70:657-62.

21. Ko YH, Kim CW, Park CS, Jang HK, Lee SS, Kim SH, et al. REAL classification of malignant lymphomas in the Republic of Korea: incidence of recently recognized entities and changes in clinicopathologic features. Hematolymphoreticular Study Group of the Korean Society of Pathologists. Revised European-American lymphoma. Cancer. 1998;83:806-12.

22. Moosdorf R. Indications, results, and pitfalls in the surgery of constrictive pericarditis. Herz. 2000;25:794-8.

23. Senni M, Redfield MM, Ling LH, Danielson GK, Tajik AJ, Oh JK. Left ventricular systolic and diastolic function after pericardiectomy in patients with constrictive pericarditis: Doppler echocardiographic findings and correlation with clinical status. J Am Coll Cardiol. 1999;33:1182-8.

24. Garcia MJ, Rodriguez L, Ares M, Griffin BP, Thomas JD, Klein AL. Differentiation of constrictive pericarditis from restrictive cardiomyopathy: assessment of left ventricular diastolic velocities in longitudinal axis by Doppler tissue imaging. J Am Coll Cardiol. 1996;27:108-14. 